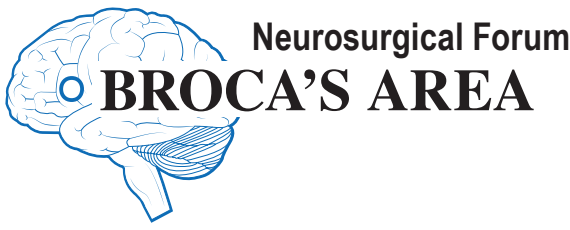

\section{Omega-3 fatty acid supplementation in severe brain trauma: case for a large multicenter trial}

\author{
Julian E. Bailes, MD, ${ }^{1,2}$ Raed Abusuwwa, DO, ${ }^{3-5}$ \\ Mohammad Arshad, DO, ${ }^{5}$ Shakeel A. Chowdhry, MD, ${ }^{1,2}$ \\ Donald Schleicher II, DO, ${ }^{3}$ Nicholas Hempeck, DO, ${ }^{3}$ \\ Yogesh N. Gandhi, MD, ${ }^{3}$ Zachary Jaffa, DO, ${ }^{3}$ \\ Faran Bokhari, MD, ${ }^{3}$ Dean Karahalios, MD, ${ }^{4}$ \\ Jeanne Barkley, MSN, ${ }^{4}$ Vimal Patel, $\mathrm{PhD},{ }^{1,2}$ and \\ Barry Sears, $\mathrm{PhD}^{6}$ \\ ${ }^{1}$ Department of Neurosurgery, NorthShore University HealthSystem, \\ Evanston; 'University of Chicago, Pritzer School of Medicine, Chicago; \\ ${ }^{3}$ Department of Neurosurgery, John H. Stroger Jr. Hospital of Cook \\ County, Chicago; ${ }^{4}$ Department of Neurosurgery, Advocate Good \\ Samaritan Hospital, Downers Grove; ${ }^{5}$ Midwestern University, Chicago \\ College of Osteopathic Medicine, Downers Grove, Illinois; and ${ }^{6}$ The \\ Inflammation Research Foundation and Zone Labs Inc., Peabody, \\ Massachusetts
}

$\mathrm{T}$ RAUMATIC brain injury (TBI), which most often occurs from falls, accidents, and automobile collisions, is a major healthcare concern, constituting a significant cause of death and disability not just in the United States but throughout the world. Motor vehicle accidents are major causes, increasing in significance in developing countries as other causes lessen. In many regions of the world there are no sophisticated and advanced treatment centers for brain injuries. TBI is considered by many authorities to be a global public health epidemic. ${ }^{1-5}$

The best strategy to avoid the detrimental short- or long-term effects of all TBIs, mild to severe, is through primary prevention or avoidance of the injury. However, once a TBI occurs, the inexorable secondary injury represents a window of opportunity for therapeutic intervention, with the potential to prevent and reduce brain damage and improve long-term patient outcomes. To date, however, promising laboratory results over the last several decades have not been translated into successful clinical trials. There are multiple reasons, with one of the most likely being that investigators have used interventions that target a single biochemical cascade rather than multiple mechanisms of injury. At present TBI appears to be too complicated for such a narrow pharmaceutical methodology. Approaches that target multiple molecular aspects of TBI-secondary injury, repair, regeneration, neurogenesis, as well as protection of the brain-are needed. One such strategy is the use of omega-3 polyunsaturated fatty acid (O3FA) supplementation. ${ }^{6-8}$

Multiple studies have shown that the O3FAs eicosapentaenoic acid (EPA) and docosahexaenoic acid (DHA) are essential for proper brain development and function. ${ }^{9,10}$ There have been numerous preclinical studies in animal models that have shown the benefits of O3FA in reducing the cellular injury and negative outcomes associated with TBI, stroke, and spinal cord injury, ${ }^{8,11-14}$ while work on the underlying mechanisms is still unfolding. ${ }^{15,16}$ Extensive human studies are lacking, and in the context of severe TBI there have been only a few individual case studies in the scientific literature in which O3FA therapy has been used and which showed positive outcomes. This inexpensive, safe, and ubiquitous dietary supplement can be readily administered by an enteral route. ${ }^{13,17,18}$ Due to the global burden and human suffering of TBI, and in lieu of no demonstrated effective pharmacological treatment, the use of O3FA presents an attractive option.

The use of high-dose O3FA for treatment of severe traumatic and hypoxic brain injuries has recently emerged and attracted interest due to the potential to mitigate the several critical steps in secondary injury. We believe that along with the numerous laboratory investigations that have demonstrated positive effects, our experience in a series of 9 patients with severe TBI in whom O3FA supplementation was provided, supports the need for a larger clinical trial to further assess the potential for improvement in outcome from severe TBI.

In our exploratory study, 9 consecutive patients who presented with severe TBI and traumatically induced coma (Glasgow Coma Scale [GCS] score $\leq 8$ ), who met inclusion criteria and were able to be consented at emer- 
gency rooms and trauma units at three level 1 trauma centers in the Chicago area, were treated with high-dose O3FA. The protocol and procedures were approved by the John H. Stroger Jr. Cook County Hospital's Institutional Review Board.

Beginning on or shortly after the day of admission, a nutritional supplement of $16.2 \mathrm{~g}$ of purified omega-3 oil was delivered daily in 2 divided doses, which was approximately $15 \mathrm{ml}$ (1 tablespoon) twice daily. The mixture was administered through a small-caliber feeding tube such as a Dobhoff tube. If the patient did not require a feeding tube, then the mixture was taken orally. This O3FA concentrate consists of a 2:1 ratio of EPA and DHA, so that participating patients received $10.8 \mathrm{~g}$ of EPA and 5.4 $\mathrm{g}$ of DHA. Every $15 \mathrm{ml}$ of omega-3 oil was mixed with 45 $\mathrm{ml}$ of Sea Health Plus, a polyphenol combination derived from various fruits and vegetables also containing seaweeds and purified aloe vera for improved emulsification of the fish oil, and providing increased antioxidant protection for the O3FAs. The mixture itself contained $4500 \mathrm{U}$ of oxygen radical absorption capacity based on its ability to absorb hydroxyl-rich free radicals (Zone Labs).

All patients were given standard treatment for severe TBI, focusing on preventing secondary injury such as brain edema, swelling, fevers, seizures, and elevated intracranial pressures (ICPs). This standard of care involved surgical evacuation of intracranial hematomas when indicated, ICP measurement, medications to reduce brain swelling, ventilator management, routine CT scans to follow the progression of the injury, and surgical procedures to minimize further brain injury (e.g., decompressive craniectomy). In this case series the experimental cohort received the same standard of care as other patients, except with the addition of the supplement.

The protocol included patients with an initial GCS score $\leq 8$ due to blunt head trauma secondary to motor vehicle collisions, falls, motorcycle accidents, or incidental trauma. Head CT images revealed hemorrhage in the majority of patients and all had ICP monitoring. Five patients underwent craniotomies to remove intracranial hematomas and for control of ICP. The length of the stay in the hospital was variable by patient due to the nature and severity of the injury.

All patients' GCS scores improved over the course of the study, suggesting that O3FA supplementation provided benefits to the neurological outcomes of severe TBI and a significant return of function. Three patients were discharged with a GCS score of 15, 5 had GCS scores of 11-12, and 1 had a GCS score of 9. The hospitalization length of stay ranged from 10 to 54 days, and the mean stay was 27.6 days. One patient died of pulmonary embolism 3 weeks following discharge from the hospital after a 54-day length of stay. Three patients were lost to followup after hospital discharge. Detailed case descriptions are provided in the Supplemental Digital Content. A summary of these 9 cases is provided in Table 1.

DHA is the most abundant long-chain polyunsaturated fatty acid in the brain and is structural in nature, constituting a key component of neuronal membranes. Numerous investigators have shown the neuroprotective properties of DHA, and EPA is considered to have antiinflammatory effects. Both of these O3FAs readily enter the brain attached to erythrocytes and cross the blood-brain barrier (BBB). Several possible mechanisms for O3FA neuroprotection have been evaluated in a variety of injury model systems, showing reduced apoptosis, oxidative stress, and mitochondrial dysfunction following O3FA treatment. ${ }^{19,20}$ O3FAs have also been shown to increase the activity of antioxidant enzymes and the mechanisms through which they may play a neuroprotective role to include reduction in excitotoxicity, modulation of calcium and potassium channels, activation of gene transcription, and formation of neuroprotectin-1 and resolvins..$^{10,18,19,21-32,35,36}$

Intracellular effects of the perturbation of calcium within brain cells and in particular mitochondria (in which calcium influx overloads the buffering capacity of mitochondria), sets in motion many deleterious biochemical events. For example calcium-dependent cascades are activated, resulting in mitochondrial damage and cytochrome c release. Ultimately, cytochrome c release can activate a caspase-3-mediated apoptotic cascade of proteolytic cleavage of cytoskeletal substrates, resulting in the axonal disconnection characteristic of traumatic axonal injury. All of the above changes can result in acute effects of TBI and are responsible for the physical, cognitive, and neurobehavioral effects of TBI, including the potential for long-term neurodegeneration.

Traumatic and anoxic brain injury should be considered as, among other pathogenetic events, a profound inflammatory event. As such it begins a proinflammatory cascade of inflammatory mediators generated from the innate immune system primarily via the microglial cells in the brain. Unfortunately, the BBB dramatically restricts the entry of antiinflammatory pharmacological agents into the brain-thus making it difficult to slow the ongoing inflammatory cascade. However, this is not the case for O3FAs such as EPA and DHA. It has been demonstrated that the transport of long-chain O6FAs, such as arachidonic acid, and long-chain O3FAs, such as EPA and DHA, lead to levels virtually equivalent in the serum and brain. ${ }^{33}$

Modulation of the inflammatory cascade by O3FAs has been proposed as a critical neuroprotective mechanism. ${ }^{15,33}$ Myelin injury and its fragmentation can result in chronic inflammation and the activation of microglia. Leukocytes can infiltrate the injured regions, and astrocytes and oligodendrocytes have an important role in buffering neurotransmitters, and in limitation of disruption of myelinization and deleterious cellular processes. In our opinion, the most active and robust compounds in this regard are O3FAs, which have been shown to have multiple putative pathways of salubrious effects on both gray and white matter. Various experiments have shown that O3FA has effects on the following aspects of brain injury and repair: antiinflammation, antioxidation, limitation of excitation, membrane protection, and neurogenesis. EPA and DHA also readily cross the $\mathrm{BBB}$, allowing immediate access to injured brain regions.

The numerous proposed mechanisms of action of O3FAs demonstrate that the biochemical pathways may be either incompletely understood or multiple in their effects of reducing traumatic axonal damage. However, the most likely manner in which these positive effects are mediated 


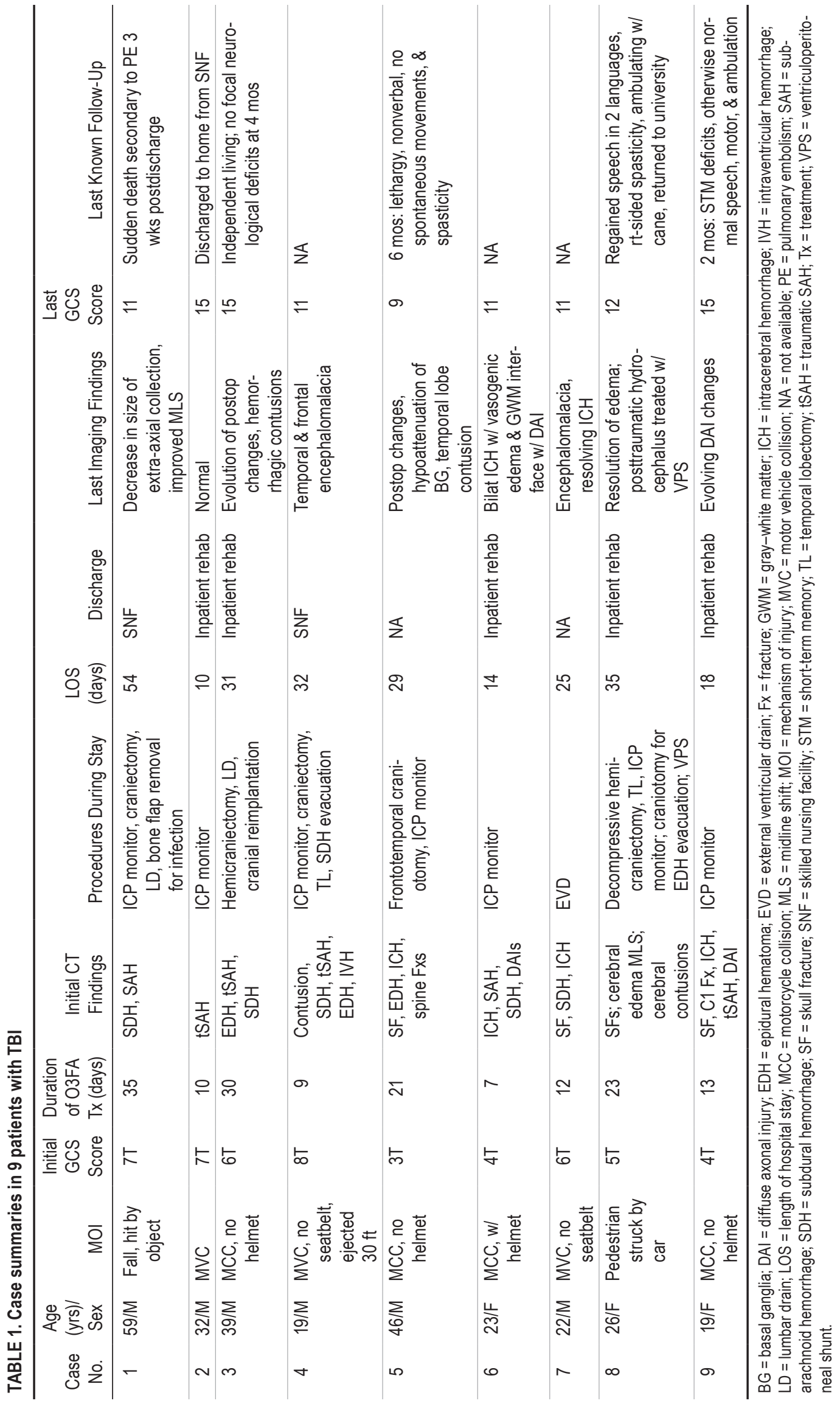


appears to involve stabilization of the cellular environment to reduce reactive oxygen species and modulate continued energy production, and production of specialized proresolution mediators that resolve inflammatory injuries. Our findings are consistent with previous research and clinical reports that have demonstrated that O3FAs are protective against cellular injury. Recently, Thau-Zuchman et al. ${ }^{34}$ administered a single-dose intravenous bolus of DHA 30 minutes after experimental brain injury and found reductions in TBI-induced neurological deficits, brain lesion size, microglial and astrocytic reaction, and oxidation, while levels of proresolving mediators were increased. In addition, there was a reduction in the accumulation of beta-amyloid precursor protein, plasma levels of neurofilament light, and axonal injury.

Although authors of multiple in vitro and animal studies and case reports have proposed various pharmacological or physiological interventions to mitigate TBI, to date no phase 3 clinical study has revealed a compound with a significant effect in reducing the morbidity and mortality of TBI. Thus the burden of TBI continues globally without a solution other than standard medical therapy and surgical intervention when indicated and possible. Our experience in 9 patients with severe TBI has shown that the use of high-dose O3FA supplementation may help to be a solution to the global health burden of TBI.

The greatest limitation of this exploratory study was the small sample size. Other study limitations include the open label, nonrandomized design and the absence of a placebo arm. Despite the limitations, a single-arm trial may be one of the few options for trials evaluating or screening therapies for TBI in which placebos may be deemed not ethical and options for controlled trials are limited. In these circumstances medical interventions use uncontrolled study data-a common approach for estimating the incremental benefit is by comparing the treatment to a "historical control"; i.e., data collected from patients who did not receive the intervention but were treated with the standard of care.

In conclusion, O3FAs are nonproprietary, ubiquitous, inexpensive, well tolerated, and easily administered orally or through an enteral feeding tube. In the absence of a substantially effective or available agent for mitigating the devastating effects of TBI worldwide, O3FA should be considered as an alternative to the present practice in many parts of the world of supportive care only for TBI victims. This is also true in underdeveloped and remote areas, as well as in war zones. There are no cost, administration, or regulatory issues that should impair the acquisition and administration of O3FA to these patients. The supplements are well tolerated with excellent safety profiles and, being essential fatty acids, should not result in allergic reactions or side effects. Our results seem to indicate a beneficial effect of O3FA even when administration is not begun immediately post-TBI. The use of these supplements can be continued long term, for months following TBI, to aid in ongoing recovery, inflammation reduction, and for neurogenesis.

Our experience has shown that O3FA supplementation may help ameliorate the multifactorial aspects of the deleterious pathophysiological changes of TBI that occur, ranging from initial and mechanical brain injury to the secondary intracellular metabolic disturbances and ongoing inflammation. Additional studies with clinical trials and prospective investigations are needed to explore further and confirm the results of our pilot data.

\section{Acknowledgments}

We acknowledge the generous support of the Inflammation Research Foundation, Peabody, MA, for providing funding and oils for this study. We thank Hannah Moulthrop, MS, for her assistance with editing, preparing figures, formatting, and submitting the manuscript for publication.

\section{References}

1. Coronado VG, McGuire LC, Sarmiento K, et al. Trends in traumatic brain injury in the U.S. and the public health response: 1995-2009. J Safety Res. 2012;43(4):299-307.

2. Dewan MC, Rattani A, Gupta S, et al. Estimating the global incidence of traumatic brain injury. J Neurosurg. 2018;130: $1-18$.

3. Humphreys I, Wood RL, Phillips CJ, Macey S. The costs of traumatic brain injury: a literature review. Clinicoecon Outcomes Res. 2013;5:281-287.

4. Ma VY, Chan L, Carruthers KJ. Incidence, prevalence, costs, and impact on disability of common conditions requiring rehabilitation in the United States: stroke, spinal cord injury, traumatic brain injury, multiple sclerosis, osteoarthritis, rheumatoid arthritis, limb loss, and back pain. Arch Phys Med Rehabil. 2014;95(5):986-995.e1.

5. Petraglia A, Maroon JC, Bailes JE. From the field of play to the field of combat: a review of medical management of concussion. Neurosurgery. 2012;70(6):1520-1533.

6. Lauritzen L, Brambilla P, Mazzocchi A, et al. DHA effects in brain development and function. Nutrients. 2016;8(1):8.

7. Lewis MD, Bailes J. Neuroprotection for the warrior: dietary supplementation with omega-3 fatty acids. Mil Med. 2011;176(10):1120-1127.

8. Mills JD, Hadley K, Bailes JE. Dietary supplementation with the omega-3 fatty acid docosahexaenoic acid in traumatic brain injury. Neurosurgery. 2011;68(2):474-481.

9. Echeverría F, Valenzuela R, Catalina Hernandez-Rodas M, Valenzuela A. Docosahexaenoic acid (DHA), a fundamental fatty acid for the brain: new dietary sources. Prostaglandins Leukot Essent Fatty Acids. 2017;124:1-10.

10. Lauritzen I, Blondeau N, Heurteaux C, et al. Polyunsaturated fatty acids are potent neuroprotectors. EMBO J. 2000;19(8):1784-1793.

11. Bailes JE, Mills JD. Docosahexaenoic acid reduces traumatic axonal injury in a rodent head injury model. J Neurotrauma. 2010;27(9):1617-1624.

12. Mills JD, Bailes JE, Sedney CL, et al. Omega-3 fatty acid supplementation and reduction of traumatic axonal injury in a rodent head injury model. J Neurosurg. 2011;114(1):77-84.

13. Roberts L, Bailes J, Dedhia H, et al. Surviving a mine explosion. J Am Coll Surg. 2008;207(2):276-283.

14. Wang X, Zhao X, Mao ZY, et al. Neuroprotective effect of docosahexaenoic acid on glutamate-induced cytotoxicity in rat hippocampal cultures. Neuroreport. 2003;14(18):24572461.

15. Calder PC. Omega-3 fatty acids and inflammatory processes: from molecules to man. Biochem Soc Trans. 2017;45(5):1105-1115.

16. Samaddar S. Effect of docosahexaenoic acid (DHA) on spinal cord injury. Adv Neurobiol. 2016;12:27-39.

17. Lewis M, Ghassemi P, Hibbeln J. Therapeutic use of omega-3 fatty acids in severe head trauma. Am J Emerg Med. 2013;31(1):273.e5-273.e8.

18. Sears B, Bailes J, Asselin B. Therapeutic uses of high-dose 
omega-3 fatty acids to treat comatose patients with severe brain injury. PharmaNutrition. 2013;1:86-89.

19. Lang-Lazdunski L, Blondeau N, Jarretou G, et al. Linolenic acid prevents neuronal cell death and paraplegia after transient spinal cord ischemia in rats. J Vasc Surg. 2003;38(3):564-575.

20. Sullivan PG, Rabchevsky AG, Waldmeier PC, Springer JE. Mitochondrial permeability transition in CNS trauma: cause or effect of neuronal cell death? J Neurosci Res. 2005;79(12):231-239.

21. Bazan NG. Cell survival matters: docosahexaenoic acid signaling, neuroprotection and photoreceptors. Trends Neurosci. 2006;29(5):263-271.

22. Belayev L, Hong SH, Menghani H, et al. Docosanoids promote neurogenesis and angiogenesis, blood-brain barrier integrity, penumbra protection, and neurobehavioral recovery after experimental ischemic Stroke. Mol Neurobiol. 2018;55(8):7090-7106.

23. Calderon F, Kim H-Y. Docosahexaenoic acid promotes neurite growth in hippocampal neurons. J Neurochem. 2004;90(4):979-988.

24. Chen X, Chen C, Fan S, et al. Omega-3 polyunsaturated fatty acid attenuates the inflammatory response by modulating microglia polarization through SIRT1-mediated deacetylation of the HMGB1/NF- $x$ B pathway following experimental traumatic brain injury. J Neuroinflammation. 2018;15(1):116.

25. Danthi SJ, Enyeart JA, Enyeart JJ. Modulation of native Ttype calcium channels by $\omega-3$ fatty acids. Biochem Biophys Res Commun. 2005;327(2):485-493.

26. Högyes E, Nyakas C, Kiliaan A, et al. Neuroprotective effect of developmental docosahexaenoic acid supplement against excitotoxic brain damage in infant rats. Neuroscience. 2003;119(4):999-1012.

27. Jump DB. Dietary polyunsaturated fatty acids and regulation of gene transcription. Curr Opin Lipidol. 2002;13(2):155164 .

28. Lonergan PE, Martin DS, Horrobin DF, Lynch MA. Neuroprotective actions of eicosapentaenoic acid on lipopolysaccharide-induced dysfunction in rat hippocampus. J Neurochem. 2004;91(1):20-29.

29. Songur A, Sarsilmaz M, Sogut S, et al. Hypothalamic superoxide dismutase, xanthine oxidase, nitric oxide, and malondialdehyde in rats fed with fish omega-3 fatty acids. Prog Neuropsychopharmacol Biol Psychiatry. 2004;28(4):693-698.

30. Sun GY, Simonyi A, Fritsche KL, et al. Docosahexaenoic acid (DHA): an essential nutrient and a nutraceutical for brain health and diseases. Prostaglandins Leukot Essent Fatty Acids. 2018;136:3-13.

31. Wu A, Ying Z, Gomez-Pinilla F. Omega-3 fatty acids supplementation restores mechanisms that maintain brain homeostasis in traumatic brain injury. $J$ Neurotrauma. 2007;24(10):1587-1595.
32. Zhu W, Chi N, Zou P, et al. Effect of docosahexaenoic acid on traumatic brain injury in rats. Exp Ther Med. 2017;14(5):4411-4416.

33. Ouellet M, Emond V, Chen CT, et al. Diffusion of docosahexaenoic and eicosapentaenoic acids through the bloodbrain barrier: an in situ cerebral perfusion study. Neurochem Int. 2009;55(7):476-482.

34. Thau-Zuchman O, Ingram R, Harvey GG, et al. A single injection of docosahexaenoic acid induces a pro-resolving lipid mediator profile in the injured tissue and a long-lasting reduction in neurological deficit after traumatic brain injury in mice. J Neurotrauma. 2020;37(1):66-79.

35. Bazan NG. Neuroprotectin D1 (NPD1): a DHA-derived mediator that protects brain and retina against cell injuryinduced oxidative stress. Brain Pathol. 2005;15(2):159-166.

36. Sarsilmaz M, Songur A, Kus I, et al. The regulatory role of dietary omega-3 essential fatty acids on oxidant/antioxidant balance in the rat hippocampus. Neurosci Res Commun. 2003;33:114-123.

\section{Disclosures}

Dr. Sears is the president of and has direct stock ownership in Zone Labs, a medical foods company that produces nutritional products including the purified O3FA concentrate used in this study. Dr. Bailes is a consultant for Trident Brands Inc., a nutritional supplement company, and is a consultant for and receives royalties from Mizuho, Inc. Dr. Chowdhry is a consultant for Medtronic, and is a member of the speaker's bureau for Portola.

\section{Supplemental Information}

\section{Online-Only Content}

Supplemental material is available with the online version of the article.

Supplemental Digital Content. https://thejns.org/doi/suppl/ 10.3171/2020.3.JNS20183.

\section{Correspondence}

Julian E. Bailes: jbailes@northshore.org.

\section{INCLUDE WHEN CITING}

Published online May 15, 2020; DOI: 10.3171/2020.3.JNS20183.

(c) 2020 The authors, CC BY-NC-ND 4.0 (http://creativecommons.org/licenses/ by-nc-nd/4.0/) 